\title{
Communication \\ Chemical Stability Analysis of Hair Cleansing Conditioners under High-Heat Conditions Experienced during Hair Styling Processes
}

\author{
Derek A. Drechsel ${ }^{1, *}$, Kevin M. Towle ${ }^{2}$, Ernest S. Fung ${ }^{3}$, Rachel M. Novick ${ }^{2}$, \\ Dennis J. Paustenbach ${ }^{4}$ and Andrew D. Monnot ${ }^{2}$ \\ 1 Cardno ChemRisk, Boulder, CO 80301, USA \\ 2 Cardno ChemRisk, San Francisco, CA 94105, USA; kevin.towle@cardno.com (K.M.T.); \\ rachel.novick@cardno.com (R.M.N.); andrew.monnot@cardno.com (A.D.M.) \\ 3 Cardno ChemRisk, Aliso Viejo, CA 92656, USA; ernest.fung@cardno.com \\ 4 Cardno ChemRisk, Jackson, WY 83001, USA; dennis.paustenbach@cardno.com \\ * Correspondence: derek.drechsel@cardno.com; Tel.: +1-720-305-5840
}

Received: 2 February 2018; Accepted: 9 March 2018; Published: 13 March 2018

\begin{abstract}
Chemical stability is a key component of ensuring that a cosmetic product is safe for consumer use. The objective of this study was to evaluate the chemical stability of commercially available hair cleansing conditioners subjected to high heat stresses from the styling processes of blow drying or straightening. Two hair cleansing conditioners were subjected to temperatures of $60^{\circ} \mathrm{C}$ and $185^{\circ} \mathrm{C}$ to simulate the use of a blow dryer or flatiron hair straightener, respectively and analyzed via Gas Chromatography-Mass Spectrometry (GC-MS), High-Performance Liquid Chromatography-UV (HPLC) and Fourier-Transform Infrared Spectroscopy (FT-IR) to capture a chemical profile of the samples. The resulting spectra from matched heated and unheated samples were compared to identify any changes in chemical composition. Overall, no differences in the spectra were observed between the heated and unheated samples at both temperatures evaluated. Specifically, no new peaks were observed during analysis, indicating that no degradation products were formed. In addition, all chemicals identified during GC-MS analysis were known listed ingredients of the products. In summary, no measurable changes in chemical composition were observed in the hair cleansing conditioner samples under high-heat stress conditions. The presented analytical methods can serve as an initial screening tool to evaluate the chemical stability of a cosmetic product under conditions of anticipated use.
\end{abstract}

Keywords: chemical analysis; stability; spectroscopy; product safety

\section{Introduction}

Personal care and cosmetic products are widely used throughout the world, with an estimated worldwide revenue of $\$ 265$ billion in 2017 [1]. Current regulatory standards, such as those issued by the Food and Drug Administration (FDA) in the United States or the European Union, do not require specific testing to demonstrate the safety of personal care and cosmetic products or ingredients [2,3]. However, trade industry associations—-such as the Personal Care Products Council (PCPC) in the United States and Cosmetics Europe in Europe-have developed resources for industry members to ensure the safety of personal care and cosmetic products. These guidelines include product stability testing, with the goal of ensuring that a "cosmetic product maintains its intended physical, chemical and microbial quality, as well as functionality and aesthetics when stored under appropriate conditions" [4].

While no standards exist for the stability testing of personal care and cosmetic products, trade associations in the United States and Europe have issued recommendations for stability testing to 
industry members [4-6]. These voluntary guidelines are intended to be general recommendations to aid manufacturers in the development of storage and shelf-life instructions for a given personal care or cosmetic product. Specific testing procedures have not been established but each manufacturer is expected to design a stability testing program that is suitable and scientifically sound for the marketed product [5]. Typical stability testing is designed to address three primary areas: (1) physical and chemical stability, including organoleptic properties (color, odor), $\mathrm{pH}$, viscosity, texture and emulsion; (2) potential for microbial contamination; and (3) the impact of packaging on the contained product. Specifically, a number of testing approaches have been developed to evaluate physical and chemical stability under predicted conditions of transport, storage and use. These include elevated temperatures, freeze-thaw cycles, light exposure and mechanical vibration. For example, thermal stability testing may be conducted under accelerated or long-term test periods ranging from one to 12 months, with products typically subjected to temperatures ranging from $25^{\circ} \mathrm{C}$ to $45^{\circ} \mathrm{C}[6]$.

Stability testing is intended to provide data to establish "an expected use life and label storage instruction" for the product under specific manufacturing and packaging conditions [4]. It should be noted that personal care and cosmetic products may be exposed to stress conditions beyond those anticipated during the transport and storage of a product. For example, physical and chemical stability testing is typically conducted under elevated temperatures up to $45^{\circ} \mathrm{C}$ for several months to simulate potential storage conditions. However, hair care products (such as shampoos or conditioners remaining on the hair or skin) may be exposed to high-heat stress from the styling processes of blow drying or straightening. Average temperatures experienced during these styling processes may reach $60^{\circ} \mathrm{C}$ or $185^{\circ} \mathrm{C}$, respectively [7-9].

Since chemical reactions are accelerated at elevated temperatures, heating processes could alter the composition of the product and subject consumers to exposure of unanticipated chemical(s). Given that standard guidelines for stability may not adequately address such conditions associated with consumer use, such as the heating of products, the purpose of this study was to evaluate the chemical stability of commercially available hair cleansing conditioner products subjected to extreme temperatures commonly experienced during the hair styling process.

\section{Methods}

\subsection{Sample Preparation}

Two samples of commercially available hair cleansing conditioners were provided directly to an analytical laboratory from the manufacturer and stored at ambient conditions until the time of analysis. All chemical testing was performed by Avomeen Analytical Services (www.avomeen.com; Ann Arbor, MI, USA). For each chemical analysis, approximately $1 \mathrm{~g}$ of product was placed into a $20 \mathrm{~mL}$ glass scintillation vial. Two vials were prepared for each sample, one remained at room temperature (unheated) while the other was heated to the indicated temperature (heated). Samples were heated in an incubation oven to the specified temperature for $15 \mathrm{~min}$ [10]. To simulate the use of a blow dryer, one sample (sample A) was heated to $60^{\circ} \mathrm{C}$. Meanwhile, the second sample (sample B) was heated to $185^{\circ} \mathrm{C}$ to simulate average temperatures experienced during use of a flatiron straightener.

Samples were removed from the oven, allowed to cool to room temperature and then analyzed to compare chemical profiles between heated and unheated samples. For High-Performance Liquid Chromatography (HPLC) and Gas Chromatography-Mass Spectrometry (GC-MS) analysis, samples were dissolved in $10 \mathrm{~mL}$ of methanol and vortexed until mixed and dissolved. Samples were filtered using a $0.45 \mu \mathrm{m}$ PTFE filter and analyzed under the conditions described below.

\subsection{HPLC-UV Analysis}

HPLC-UV analysis was performed using an Agilent 1100 system with a diode-array detector (DAD) using wavelengths of $210 \mathrm{~nm}, 230 \mathrm{~nm}, 254 \mathrm{~nm}$ and $280 \mathrm{~nm} .10 \mu \mathrm{L}$ of sample was injected and separated using $5 \mu \mathrm{m}$ Kinetex Luna C18 $4.6 \mathrm{~mm} \times 150 \mathrm{~mm}$ column at $30^{\circ} \mathrm{C}$. The mobile phase 
consisted of $85 \%$ deionized water and $15 \%$ acetonitrile for $20 \mathrm{~min}$, followed by $5 \%$ deionized water and $95 \%$ acetonitrile for $10 \mathrm{~min}$ and finally $85 \%$ deionized water and $15 \%$ acetonitrile for $15 \mathrm{~min}$ at a constant flow rate of $1.0 \mathrm{~mL} / \mathrm{min}$. All injections were run in triplicate.

\subsection{GC-MS Analysis}

GC-MS analysis was performed using an Agilent $6890 \mathrm{~N}$ with the MSD 5973 system. For matched samples subject to heating at $60^{\circ} \mathrm{C}$, a $0.25 \mu \mathrm{m}$ HP- $530 \mathrm{~m} \times 0.32 \mathrm{~mm}$ column was used, while a $1 \mu \mathrm{m}$ DB-5 $30 \mathrm{~m} \times 0.25 \mathrm{~mm}$ column was used for matched samples subject to heating at $185^{\circ} \mathrm{C}$. All other parameters described below were the same for all tested samples. The sample injection volume was $1 \mu \mathrm{L}$ at $250{ }^{\circ} \mathrm{C}$. Column flow was constant at a rate of of $1.0 \mathrm{~mL} / \mathrm{min}$. The GC oven temperature was programmed at $50{ }^{\circ} \mathrm{C}$ and increased to $300{ }^{\circ} \mathrm{C}$ at a linear rate of $15^{\circ} \mathrm{C} / \mathrm{min}$. Temperatures of the transfer line, quadruple and ion source were $280^{\circ} \mathrm{C}, 150{ }^{\circ} \mathrm{C}$ and $230{ }^{\circ} \mathrm{C}$, respectively. MS analysis conditions were set to detect a mass range of 200-800 Da. All injections were run in triplicate.

\subsection{FT-IR Analysis}

For Fourier-Transform Infrared Spectroscopy (FT-IR) analysis, after heating and cooling to room temperature, samples were placed in a $-40{ }^{\circ} \mathrm{C}$ freezer for two hours and were subsequently dried for two days at $-80^{\circ} \mathrm{C}$ using a lyophilizer. Dried samples were analyzed directly using a Perkin Elmer Spectrum 65 FT-IR. Scans were performed over the spectral range of $4000 \mathrm{~cm}^{-1}$ to $600 \mathrm{~cm}^{-1}$, at a resolution of $4 \mathrm{~cm}^{-1}$.

\section{Results}

\subsection{HPLC-UV}

Several compounds were detected in the HPLC chromatograms of the matched unheated and heated samples for both $60^{\circ} \mathrm{C}$ and $185^{\circ} \mathrm{C}$ at all wavelengths examined. Representative chromatograms for matched heated and unheated samples examined at $254 \mathrm{~nm}$ are presented in Figure 1A,B. The number of compounds, retention time and peak area for each sample were found to be reproducible based on triplicate analysis. No differences were observed in the matched heated and unheated samples for both the blow dryer and hair straighter scenarios. Specifically, all of the peaks in the chromatograms for the matched unheated and heated samples had the same retention times and any difference in peak height or area under the curve for the compounds in the matched unheated and heated samples was less than $12 \%$.

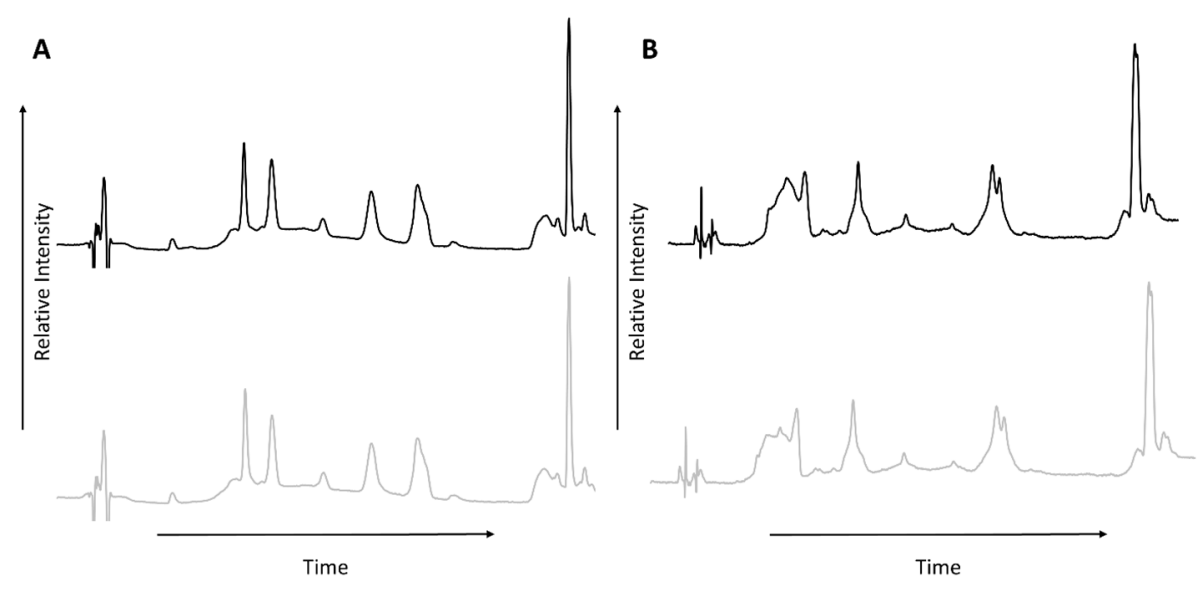

Figure 1. Representative High-Performance Liquid Chromatography-UV (HPLC-UV) chromatograms of matched unheated (black) and heated (grey) samples with heating to $60^{\circ} \mathrm{C}(\mathbf{A})$ or $185^{\circ} \mathrm{C}(\mathbf{B})$ at $254 \mathrm{~nm}$. 


\subsection{GC-MS}

Nine compounds were identified in sample A in the matched unheated and $60{ }^{\circ} \mathrm{C}$ heated analyses (Figure 2A). Several peaks in the chromatograms were also found in the blank injection samples and were therefore not labeled and disregarded from the analysis. The number of compounds, retention time and peak height for each sample were found to be reproducible based on triplicate analysis. No differences were observed in the number of compounds, retention times or peak height between the unheated and heated samples. Chemical compounds for the nine peaks were identified through a known spectra library (Table 1).

A

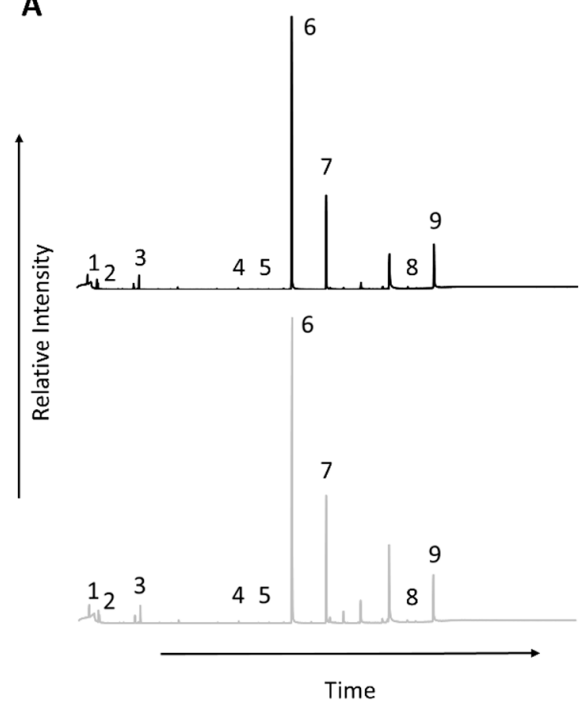

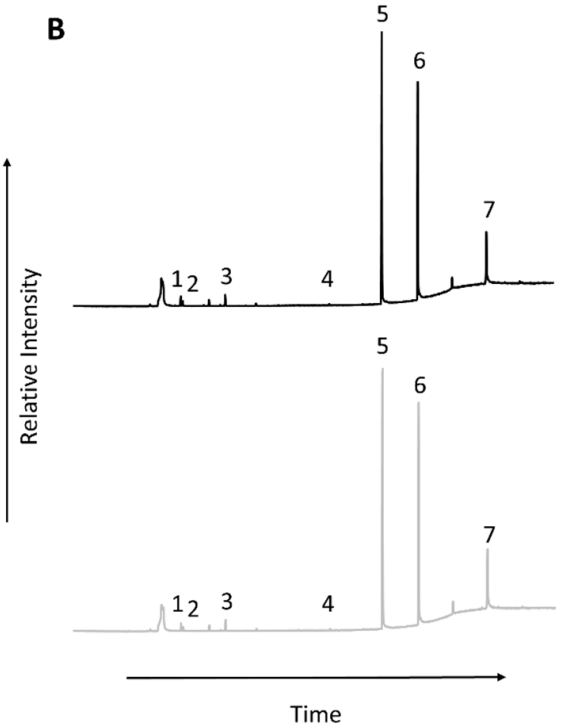

Figure 2. Representative total ion chromatograms of matched unheated (black) and heated (grey) samples with heating to $60^{\circ} \mathrm{C}(\mathbf{A})$ or $185^{\circ} \mathrm{C}$ (B). Numbered peaks were those identified by comparison to a known spectra library (see text for details). Unlabeled peaks in the chromatograms were also found in blank injections and were thus not subject to identification.

Table 1. Compounds identified in hair cleansing conditioner products during Gas ChromatographyMass Spectrometry (GC-MS) analysis.

\begin{tabular}{ccc}
\hline Peak Number from Total Ion Chromatogram & Sample A $\left(\mathbf{6 0}{ }^{\circ} \mathbf{C}\right)$ & Sample B (185 $\left.{ }^{\circ} \mathbf{C}\right)$ \\
\hline $\mathbf{1}$ & D-Limonene & D-Limonene \\
Eucalyptol & Eucalyptol & (Menthanol) \\
$\mathbf{2}$ & 1-Methyl-4-(1-methylethyl)-cyclohexanol & 1-Methyl-4-(1-methylethyl)-cyclohexanol \\
4 & (Menthanol) & Diethyl phthalate \\
$\mathbf{5}$ & Diethyl phthalate & 1-Hexadecanol (Cetyl alcohol) \\
$\mathbf{6}$ & Dexpanthenol & 1-Octadecanol (Stearyl alcohol) \\
7 & 1-Hexadecanol (Cetyl alcohol) & Dimantine \\
$\mathbf{8}$ & 1-Octadecanol (Stearyl alcohol) & - \\
\hline
\end{tabular}

Seven compounds were identified in sample B in the matched unheated and $185{ }^{\circ} \mathrm{C}$ heated analyses (Figure 2B). The number of compounds, retention time and peak heights for each sample were found to be reproducible based on triplicate analysis. No differences were observed in terms of peak number, retention time or peak area between the unheated and heated samples. The identified chemical compounds are presented in Table 1. All of the chemicals identified in the GC-MS analyses for both products were listed as ingredients or components of ingredients in the respective commercially available cleansing conditioners. 


\subsection{FT-IR}

The FT-IR spectra of the matched unheated and heated samples are shown in Figure 3. No significant changes were observed between the matched unheated and heated samples upon heating to $60^{\circ} \mathrm{C}$ or $185^{\circ} \mathrm{C}$. There were no additional peaks or shifts in absorption, nor was there any evidence of loss of absorption upon heating.

A

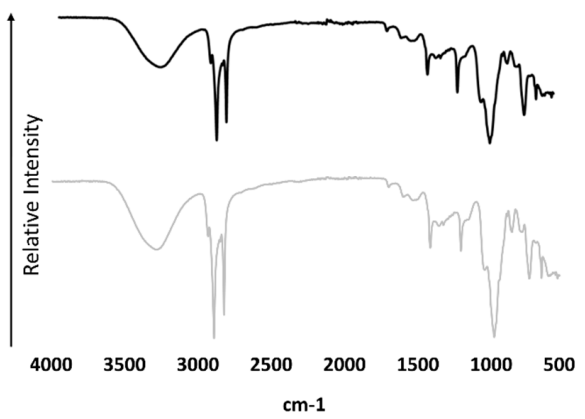

B

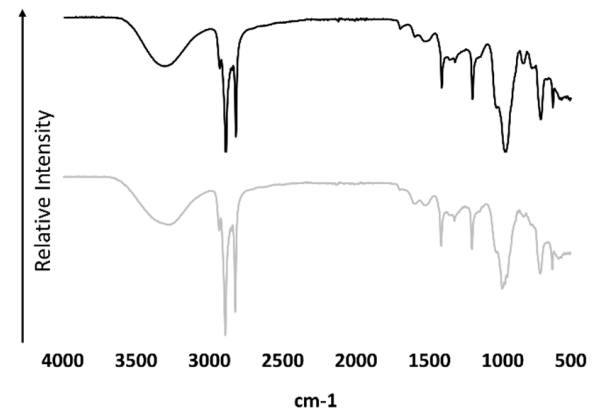

Figure 3. Representative Fourier-Transform Infrared Spectroscopy (FT-IR) spectra of matched unheated (black) and heated (grey) samples with heating to $60^{\circ} \mathrm{C}(\mathrm{A})$ or $185^{\circ} \mathrm{C}(\mathrm{B})$.

\section{Discussion}

This study demonstrates the use of analytical methods to examine the chemical stability of a personal care product under simulated conditions of use. Specifically, we subjected samples from a commercial hair cleansing conditioner product line to temperature stresses experienced during the hair styling processes of blow drying or hair straightening. No changes in the chemical composition of matched unheated and heated samples were observed via HPLC, GC-MS and FT-IR analyses. Specifically, no other products were observed after heating samples to $60^{\circ} \mathrm{C}$ or $185^{\circ} \mathrm{C}$, indicating that no detectable degradation products were formed following high temperature stress. This approach represents a form of stability testing that can be adapted to evaluate specific stressors that may be encountered during the anticipated use of a particular personal care or cosmetic product.

This analysis provides an example of examining the chemical stability of a hair cleansing conditioner subjected to temperatures outside the bounds of standard stability testing guidelines due to the increased temperatures experienced during hair styling processes. These extreme temperature variations are examples of conditions that can be incorporated on a product-specific basis when developing a stability testing program.

Ensuring the stability of ingredients used in personal care and cosmetic products under situations of high heat stress may be necessary to assure that consumers are not exposed to unforeseen chemicals, which could result in adverse health effects. Ingredients such as fragrance chemicals, preservatives and "natural" products, including botanicals and essential oils, have been identified as allergens with the potential for inducing skin sensitization [11-13]. Specifically, several chemicals found in fragrances and botanicals are subject to oxidation that may impart allergenic potential to the resulting degradation product. Examples of this include the auto-oxidation products resulting from degradation of commonly used cosmetic ingredients, such as geraniol, linalool, limonene and constituents of lavender oil [14-18].

Temperature is known to be an important determinant in the stability of chemicals and oxidative degradation may be enhanced with increasing temperature. Specifically, the Arrhenuis equation demonstrates that chemical reaction rate increases with increasing temperature $[3,19,20]$. While others have examined factors of product stability among personal care and cosmetic products (including storage temperature, ethanol, $\mathrm{pH}$ and UV light), this is the first known study to evaluate whether the stability of hair care products is impacted by temperature variations experienced during the hair styling process [21-23]. 
Analytical methods, including HPLC and mass spectrometry, have been used with increasing frequency in the field of cosmetics to assess the chemical nature of product formulation and potential degradation products [24,25]. These approaches can be used to achieve a high degree of specificity and sensitivity lacking in less sophisticated methods. The methods applied in this analysis were intended to provide a screening-level assessment of potential chemical changes resulting from exposure to extreme temperature variations. While the methods utilized in this analysis were intended to detect a variety of chemicals present in hair cleansing conditioners (volatile vs. non-volatile, organic vs. non-organic), it was not designed to identify all potential compounds and their concentrations. Based on a product's formulation and any particular concerns about any individual ingredient or chemical, more specific procedures and analyses beyond the screening-level approach discussed here can be developed as part of a stability testing program. For example, several authors have developed the means to detect chemicals capable of inducing skin sensitization resulting from the oxidation of fragrance materials [26,27].

While this analysis was intended to simulate the impact of high temperature stress of hair-care products, it is important to recognize that it is not completely representative of conditions experienced by the consumer. Consumer product usage surveys have reported that the mean amount of shampoo and conditioner product applied per application is $11 \mathrm{~g}$ and $13 \mathrm{~g}$, respectively [28,29]. However, only one percent of the product is expected to remain on the hair and skin following washing and rinsing [30]. Therefore, it is likely that any product remaining on the hair and skin would form a thin film, with an increased surface area to volume ratio than the form of the product examined in the current study. Under these conditions, heating may lead to changes in temperature distribution, increased loss of volatile chemicals through vaporization, or increased oxidation reactions. Future analyses may seek to address such issues.

\section{Conclusions}

Overall, this analysis provides evidence that subjecting a commercial hair cleansing conditioner to temperatures which may be experienced during the hairstyling process does not result in detectable degradation of the product. These results suggest that consumers would not be exposed to any unforeseen chemical constituents that could be formed as a result of product degradation from high-heat stress. The presented analytical methods can serve as an initial screening tool to evaluate chemical stability of a personal care or cosmetic product under conditions of anticipated use.

Author Contributions: All authors (Derek A. Drechsel, Kevin M. Towle, Ernest S. Fung, Rachel M. Novick, Dennis J. Paustenbach, and Andrew D. Monnot) conceived and designed the experiments; Avomeen Analytical Services performed the experiments; Derek A. Drechsel, Kevin M. Towle, and Andrew D. Monnot analyzed the data; Derek A. Drechsel, Kevin M. Towle, and Andrew D. Monnot wrote the paper.

Conflicts of Interest: Authors Derek A. Drechsel, Kevin M. Towle, Ernest S. Fung, Rachel M. Novick, Dennis J. Paustenbach and Andrew D. Monnot are employed by Cardno ChemRisk, a consulting firm that provides scientific advice to the government, corporations, law firms and various scientific/professional organizations. Cardno ChemRisk has been engaged by WEN by Chaz Dean, Inc. (WCD), which produces personal care products, including the product examined in this study. This paper was prepared and written exclusively by the authors without review or comment by any outside entity. It is possible that this work will be relied upon in litigation. Funding for the research and preparation of this article was provided by WCD.

\section{References}

1. Yeomans, M. Global Beauty Market to Reach $\$ 265$ Billion in 2017 Due to an Increase in GDP. Available online: https:/ / www.cosmeticsdesign.com/Article/2012/11/07/Global-beauty-market-to-reach265-billion-in-2017-due-to-an-increase-in-GDP (accessed on 22 January 2018).

2. Food and Drug Administration (FDA). FDA Authority over Cosmetics: How Cosmetics Are Not FDA-Approved, but Are FDA-Regulated. Available online: https://www.fda.gov/Cosmetics / GuidanceRegulation/LawsRegulations/ucm074162.htm (accessed on 27 November 2017). 
3. Regulation EU No. 1223/2009 of the European Parliament and of the Council of 30 November 2009 on Cosmetic Products (Recast) (Text with EEA Relevance). L 342/59. Available online: http:/ / eur-lex.europa. eu/legal-content/EN/TXT/?uri=CELEX:02009R1223-20160812\&from=EN (accessed on 6 March 2018).

4. Personal Care Products Council (PCPC). Guidelines for Industry: The Stability Testing of Cosmetics; Personal Care Products Council: Washington, DC, USA, 2011; p. 1.

5. Cosmetic, Toiletry and Fragrance Association (CTFA); Colipa. Guidelines on Stability Testing of Cosmetic Products. 03/094-MC. March 2004; Cosmetics Europe, Personal Care Association: Brussels, Belgium, 2004.

6. International Federation of Societies of Cosmetic Chemists (IFSCC). Monograph Number 2: The Fundamentals of Stability Testing; Micelle Press: Weymouth, UK, 1992.

7. Lee, Y.; Kim, Y.D.; Hyun, H.J.; Pi, L.Q.; Jin, X.; Lee, W.S. Hair shaft damage from heat and drying time of hair dryer. Ann. Dermatol. 2011, 23, 455-462. [CrossRef] [PubMed]

8. Breuning, E.E.; Papini, R.P. Hair straighteners: A significant burn risk. Burns 2008, 34, 703-706. [CrossRef] [PubMed]

9. Dussaud, A.; Rana, B.; Lam, H.T. Progressive hair straightening using an automated flat iron: Function of silicones. J. Cosmet. Sci. 2013, 64, 119-131. [PubMed]

10. Harding, E. How Much Time Do You Really Spend Doing Your Hair, Ladies? Answer: Ten Days a Year! Available online: http:/ / www.dailymail.co.uk/femail/article-2250701/How-time-really-spend-doing-hairladies-Answer-Ten-days-year.html (accessed on 8 September 2016).

11. Bickers, D.R.; Calow, P.; Greim, H.A.; Hanifin, J.M.; Rogers, A.E.; Saurat, J.H.; Sipes, I.G.; Smith, R.L.; Tagami, H. The safety assessment of fragrance materials. Regul. Toxicol. Pharmacol. 2003, 37, 218-273. [CrossRef]

12. Scientific Committee on Consumer Safety (SCCS). Opinion on the Mixture of 5-Chloro-2-Methylisothiazolin3(2H)-One and 2-Methylisothiazolin-3(2H)-One. COLIPA n ${ }^{\circ}$ P56. SCCS/1238/09.

13. Antignac, E.; Nohynek, G.J.; Re, T.; Clouzeau, J.; Toutain, H. Safety of botanical ingredients in personal care products/cosmetics. Food Chem. Toxicol. 2011, 49, 324-341. [CrossRef] [PubMed]

14. Opdyke, D.L.J. Monographs on fragrance raw materials. Food Cosmet. Toxicol. 1975, 13, 683-923. [CrossRef]

15. Skold, M.; Borje, A.; Matura, M.; Karlberg, A.T. Studies on the autoxidation and sensitizing capacity of the fragrance chemical linalool, identifying a linalool hydroperoxide. Contact Dermat. 2002, 46, 267-272. [CrossRef]

16. Hagvall, L.; Backtorp, C.; Svensson, S.; Nyman, G.; Borje, A.; Karlberg, A.T. Fragrance compound geraniol forms contact allergens on air exposure. Identification and quantification of oxidation products and effect on skin sensitization. Chem. Res. Toxicol. 2007, 20, 807-814. [CrossRef] [PubMed]

17. Skold, M.; Hagvall, L.; Karlberg, A.T. Autoxidation of linalyl acetate, the main component of lavender oil, creates potent contact allergens. Contact Dermat. 2008, 58, 9-14. [CrossRef] [PubMed]

18. Brared Christensson, J.; Forsstrom, P.; Wennberg, A.M.; Karlberg, A.T.; Matura, M. Air oxidation increases skin irritation from fragrance terpenes. Contact Dermat. 2009, 60, 32-40. [CrossRef] [PubMed]

19. Peleg, M.; Normand, M.D.; Corradini, M.G. The Arrhenius equation revisited. Crit. Rev. Food Sci. Nutr. 2012, 52, 830-851. [CrossRef] [PubMed]

20. Schwarzenbach, R.P.; Gschwend, P.M.; Imboden, D.M. Environmental Organic Chemistry, 2nd ed.; J. Wiley: Hoboken, NJ, USA, 2003.

21. Huang, Y.; Lenaghan, S.C.; Xia, L.; Burris, J.N.; Stewart, C.N., Jr.; Zhang, M. Characterization of physicochemical properties of ivy nanoparticles for cosmetic application. J. Nanobiotechnol. 2013, 11, 3. [CrossRef] [PubMed]

22. Berthele, H.; Sella, O.; Lavarde, M.; Mielcarek, C.; Pense-Lheritier, A.M.; Pirnay, S. Determination of the influence of factors (ethanol, $\mathrm{pH}$ and $\mathrm{a}(\mathrm{w})$ ) on the preservation of cosmetics using experimental design. Int. J. Cosmet. Sci. 2014, 36, 54-61. [CrossRef] [PubMed]

23. Rodrigues, F.; Sarmento, B.; Amaral, M.H.; Oliveira, M.B. Exploring the antioxidant potentiality of two food by-products into a topical cream: Stability, in vitro and in vivo evaluation. Drug Dev. Ind. Pharm. 2016, 42, 880-889. [CrossRef] [PubMed]

24. De Oliveira, D.N.; Delafiori, J.; Ferreira, M.S.; Catharino, R.R. In vitro evaluation of Sun Protection Factor and stability of commercial sunscreens using mass spectrometry. J. Chromatogr. B Anal. Technol. Biomed. Life Sci. 2015, 988, 13-19. [CrossRef] [PubMed] 
25. Cattley, K.; Duracher, L.; Camattari, P.; Mavon, A.; Grooby, S. Pre-clinical formulation screening, development and stability of acetyl aspartic acid for cosmetic application. Int. J. Cosmet. Sci. 2015, 37 (Suppl. 1), $28-33$. [CrossRef] [PubMed]

26. Kern, S.; Dkhil, H.; Hendarsa, P.; Ellis, G.; Natsch, A. Detection of potentially skin sensitizing hydroperoxides of linalool in fragranced products. Anal. Bioanal. Chem. 2014, 406, 6165-6178. [CrossRef] [PubMed]

27. Christensson, J.B.; Hagvall, L.; Karlberg, A.-T. Fragrance allergens, overview with a focus on recent developments and understanding of abiotic and biotic activation. Cosmetics 2016, 3, 19. [CrossRef]

28. Loretz, L.; Api, A.M.; Barraj, L.; Burdick, J.; Davis, D.A.; Dressler, W.; Gilberti, E.; Jarrett, G.; Mann, S.; Pan, Y.L.; et al. Exposure data for personal care products: Hairspray, spray perfume, liquid foundation, shampoo, body wash and solid antiperspirant. Food. Chem. Toxicol. 2006, 44, 2008-2018. [CrossRef] [PubMed]

29. Loretz, L.J.; Api, A.M.; Babcock, L.; Barraj, L.M.; Burdick, J.; Cater, K.C.; Jarrett, G.; Mann, S.; Pan, Y.H.L.; Re, T.A.; et al. Exposure data for cosmetic products: Facial cleanser, hair conditioner and eye shadow. Food Chem. Toxicol. 2008, 46, 1516-1524. [CrossRef] [PubMed]

30. Scientific Committee on Consumer (SCCS). The SCCS Notes of Guidance for the Testing of Cosmetic Ingredients and Their Safety Evaluation, 9th Revision. SCCS/1564/15. Revised Version of 25 April 2016. Available online: http:/ / ec.europa.eu/health/scientific_committees/consumer_safety/docs/sccs_O_190.pdf (accessed on 12 December 2017).

(C) 2018 by the authors. Licensee MDPI, Basel, Switzerland. This article is an open access article distributed under the terms and conditions of the Creative Commons Attribution (CC BY) license (http:// creativecommons.org/licenses/by/4.0/). 